\title{
Fuzzy Comprehensive Evaluation of Aged Community Home-Care Service Quality Based on AHP
}

\author{
Xiaoqin $\mathrm{Li}^{1, \mathrm{a}}$, Jing Wang ${ }^{1, \mathrm{~b}}$ \\ ${ }^{1}$ School of Law, Jiangxi Science and Technology Normal University, Nanchang 330038, China; \\ axq527@126.com, ${ }^{\mathrm{b}} 16837063 @ q q . c o m$
}

Keywords: aged care service, service quality, AHP, fuzzy comprehensive evaluation

\begin{abstract}
Aged care is becoming serious social problem in our world, aged community home-care is an important part and informatics is an inevitable trend in the future. How to evaluate the quality of community home-care services for elderly objectively and impartially is of vital importance to the construction of the community home-care system. Using the models developed from AHP and fuzzy comprehensive evaluation, a quantitative evaluation index system for aged community home-care services is constructed in this paper from five aspects of living care, medical and nursing care, safety and security guarantee, spiritual consolation and social participation. According to the model, the service quality in 20 communities in Nanchang was evaluated. The results show that the average level of service quality is between "general" and "good". This shows that the construction of community home aged care in Nanchang has achieved remarkable achievement, but there is still an urgent need for a further escalation and improvement.
\end{abstract}

\section{1 . Introduction}

In recent years, relatively few academic works has been done on the evaluation of community home aged care service. Most of the researchers analyze the various factors that affect the community home care service from the view of the requirements aged care and construct the relevant index system according to the statistics analysis. And most of the service quality indicators are hard to quantitative due to its vague of meaning and extent. Zuo Jianyi ${ }^{[2]}$ proposed a quality evaluation method from two aspects of community home aged care system and the satisfaction of the consumer. Zhang xiaoyi, etc $^{[3]}$ constructed a community home care quality evaluation mode from clean-aid, meal-aid, medical-aid and amusement-aid four dimensions. Liao Chuhui,etc ${ }^{[4]}$ pointed out that the operational cost, the sound level of function of the aged care service and confidence of fit between the real spiritual demands and their real culture life are the main influence factors for the aged care service quality. This paper attempt to combine the AHP and FCE to establish an aged care service quality evaluation mode based on the analysis of the research achievements at home and abroad, from the view of the feeling of the old people who had received the aged care services in Nanchang city.

The rest of this paper is organized as follows. In Section II, we introduce the construction of the community home aged care service quality index system. We analyze and evaluate the service quality according to the proposed index system based on the AHP and FCE in detail in Section III. Subsequently, the results and its analysis are presented in Section IV. Finally, we give conclusions to our works in Section V.

\section{Community home aged care service quality index system}

To determine the community home aged care services quality indicators, the paper refers to the demands for aged care services from "Social pension system construction plan(2011-2015)", and selects the various already launched services, then integrates with the opinions of experts and the data gathered from the questionnaire survey. The final service quality evaluation table includes five criteria layer indexes and 25 indicator layer indexes. The first criteria layer index is "living care". "Living care" is one of the most fundamental and important service for the aged, which includes 
seven sub-indexes: full-time housekeeper services, aged nursery services, hourly worker services, milk delivery service, laundry services, elderly meal services, indoor cleaning services. The second criteria layer index is "medical and nursing care", which includes five sub-indexes: home sick-bed services、daily professional nursing services、 postoperative rehabilitation nursing service、rational use of drugs and home safety guidance services、Acute disease nursing services. The third criteria layer index is "safety and security guarantee", which includes five sub-indexes: home appliance repair services, liquefied gas replacement services, drains dredging services, hydropower maintenance and repair services and elderly special equipment services. The forth criteria layer index is "spiritual consolation", which includes chatting services, reading services, escort services and social meeting for single senior citizens. And the fifth criteria layer index is "social participation", which includes tour and sight-seeing services, senior citizen college service, senior citizen association services, voluntary labor organization services. All these are illustrated in table 1 .

Table1. Community home care service quality evaluation system

\begin{tabular}{|c|c|c|}
\hline Targetlayers & Criterion layer & Index layer \\
\hline \multirow{25}{*}{5} & \multirow{7}{*}{ Living care(B1) } & full-time housekeeper services (C11) \\
\hline & & aged nursery services (C12) \\
\hline & & hourly worker services (C13) \\
\hline & & milk delivery service (C14) \\
\hline & & laundry services (C15) \\
\hline & & elderly meal services (C16) \\
\hline & & indoor cleaning services (C17) \\
\hline & \multirow{5}{*}{$\begin{array}{c}\text { medical and nursing } \\
\text { care (B2) }\end{array}$} & home sick-bed services (C21) \\
\hline & & daily professional nursing services (C22) \\
\hline & & postoperative rehabilitation nursing service (C23) \\
\hline & & rational use of drugs and home safety guidance services(C24) \\
\hline & & Acute disease nursing services(C25) \\
\hline & \multirow{5}{*}{$\begin{array}{l}\text { safety and security } \\
\text { guarantee (B3) }\end{array}$} & home appliance repair services (C31) \\
\hline & & liquefied gas replacement services (C32) \\
\hline & & drains dredging services (C33) \\
\hline & & hydropower maintenance and repair services (C34) \\
\hline & & elderly special equipment services (C35) \\
\hline & \multirow{4}{*}{$\begin{array}{l}\text { spiritual consolation } \\
\text { (B4) }\end{array}$} & chatting services (C41) \\
\hline & & reading services (C42) \\
\hline & & escort services (C43) \\
\hline & & social meeting for single senior citizens services(C44) \\
\hline & \multirow{4}{*}{$\begin{array}{l}\text { social participation } \\
\text { (B5) }\end{array}$} & tour and sight-seeing services (C51) \\
\hline & & senior citizen college service (C52) \\
\hline & & senior citizen association services (C53) \\
\hline & & voluntary labor organization services (C54) \\
\hline
\end{tabular}

\section{Fuzzy comprehensive evaluation based on AHP}

Due to the evaluation index and its importance is determined by the subjective judgment, evaluating the reliability of the results is not high. Therefore, it can be by using the AHP ${ }^{[5,6,7]}$ and fuzzy comprehensive evaluation $^{[8,9]}$ of the combination method to carry on the evaluation, which can improve the accuracy and reliability of the evaluation results, and the evaluation of the implementation of the computer.

\subsection{Basic thought}

The main ideas and technical routes as shown in Figure 1: 


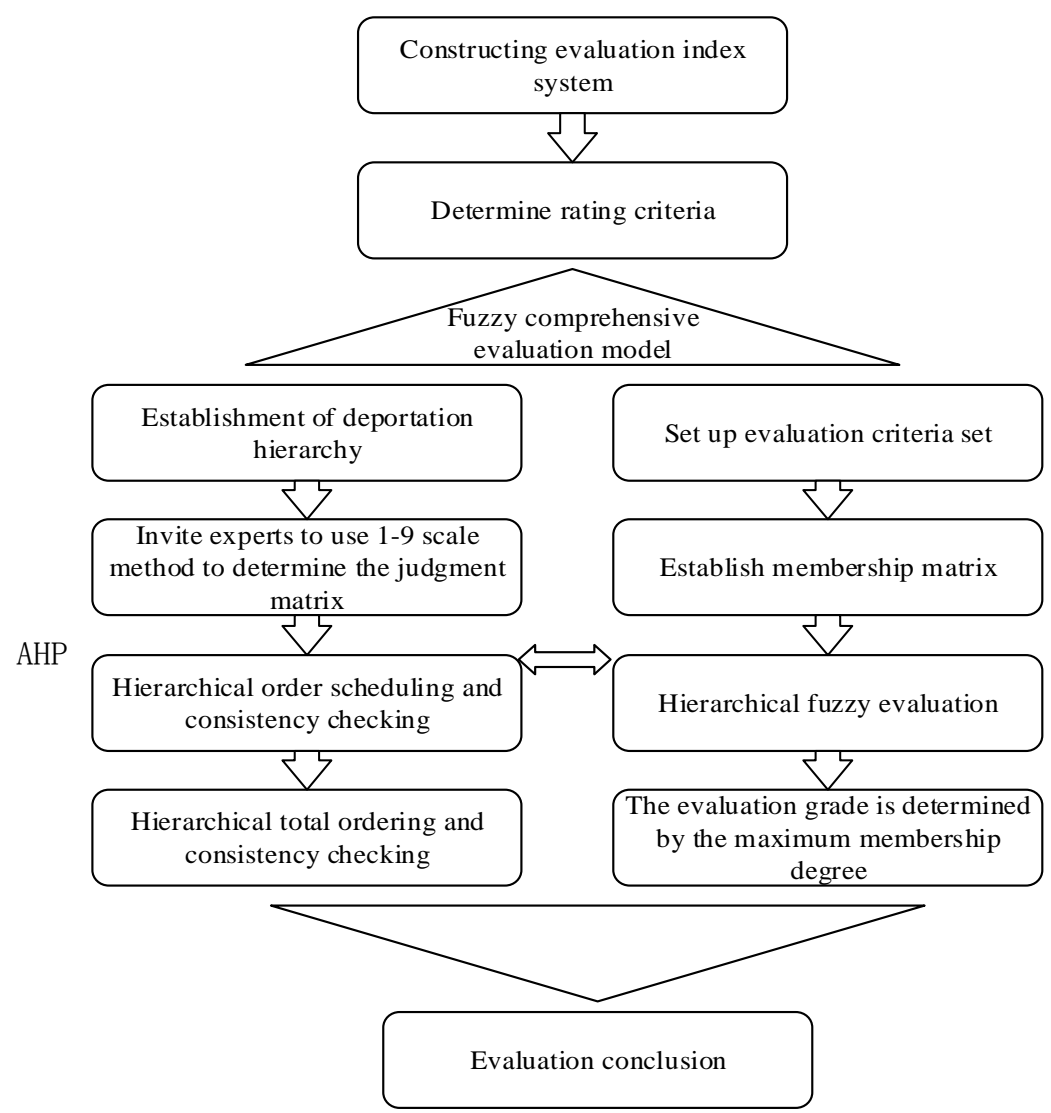

FCEM

Fig 1.Fuzzy comprehensive evaluation model based on Analytic Hierarchy Process

\subsection{Analytic hierarchy process to determine the weight}

We study the problems in advance and to draw up standard questionnaires, to the Nanchang Station 12 experts and scholars of questionnaire survey, the respondents to answer according to certain rules into the corresponding quantitative data.

\subsubsection{Setting judgment matrix according to judgment value}

In the model, 5 criteria layer index and 25 index layers are used to divide the method into 6 matrix, the judgment matrix of the quasi stratified layer is as follows. The judgment matrix of the criterion layer is:

$$
B=\left[\begin{array}{lllll}
1.000 & 1.000 & 2.000 & 1.000 & 2.000 \\
1.000 & 1.000 & 2.000 & 3.000 & 2.000 \\
0.500 & 1.000 & 1.000 & 1.000 & 1.000 \\
1.000 & 0.333 & 1.000 & 2.000 & 0.171 \\
0.500 & 0.500 & 1.000 & 0.500 & 1.000
\end{array}\right]
$$

\subsubsection{Consistency check}

A case study on the consistency of the judgment matrix of the criterion layer. In order to check the consistency of the judgment matrix, the consistency index should be calculated:

$$
C I=\frac{\lambda_{A}{ }^{\max }-n}{n-1}=\frac{5.3512-5}{5-1}=0.0878
$$

The random consistency ratio:

$$
C R=\frac{C I}{R I}=\frac{0.0878}{1.12}=0.0784<0.10
$$

It is indicated that the matrix B can be used as the weight by the consistency test. 


\subsection{Determine the membership matrix}

The expert scoring method combined with the field survey, the use of qualitative indicators to determine the degree of membership matrix. For example, invited 12 experts on " hourly worker services (C13)" the grade evaluation, four experts think "excellent", three experts believe in "good", five experts believe that the "general".Each level of experts is divided by the total number of experts "excellent" membership degree is "0.333", "good" membership degree is "0.25", the membership degree of the "general" is "0.417", get a summary of " hourly worker services (C13)" the membership matrix is [0.667 0.250 .08300 ].The method of determining the membership degree of other qualitative indicators is similar to that of the method.

\subsection{Hierarchical fuzzy evaluation}

Based on the hierarchical fuzzy evaluation, the results of the fuzzy evaluation of each index of the B layer and the result of the evaluation of the weight of the target layer are calculated.

\subsubsection{First level comprehensive evaluation}

Respectively, the fuzzy comprehensive evaluation matrix of each index is calculated after a summary of the results of the first level evaluation results, as shown in Table 2.

Table 2.Evaluation model first level evaluation results

\begin{tabular}{|c|c|c|c|c|c|}
\hline \multirow{2}{*}{ Criterion layer B } & \multicolumn{5}{|c|}{ Service quality evaluation results } \\
\cline { 2 - 5 } & Excellent & Good & General & Poor & Very poor \\
\hline Living care(B1) & 0.3473 & 0.2747 & 0.2955 & 0.0579 & 0.0246 \\
\hline medical and nursing care (B2) & 0.1950 & 0.5458 & 0.1733 & 0 & 0.0859 \\
\hline safety and security guarantee (B3) & 0.0373 & 0.2430 & 0.7000 & 0.0197 & 0 \\
\hline spiritual consolation (B4) & 0.0567 & 0.3333 & 0.5833 & 0.0266 & 0 \\
\hline social participation (B5) & 0.3550 & 0.3871 & 0.2317 & 0.0262 & 0 \\
\hline
\end{tabular}

\subsubsection{Second level comprehensive evaluation}

The results of the first level evaluation in a comprehensive evaluation and the weight of the $\mathrm{B}$ relative to the A, the second final evaluation results, which are shown in Table 3.

Table 3. Evaluation model second levels of evaluation results

Aged card service quality

\begin{tabular}{|c|c|c|c|c|}
\hline Excellent & Good & General & Poor & Very poor \\
\hline 0.2011 & 0.3754 & 0.3665 & 0.0248 & 0.0320 \\
\hline
\end{tabular}

\section{Results and analysis}

According to the calculation of the value of the standard layer A relative to the target layer B weight, we can get the relative importance of the various factors of standard layer. In terms of aged care services, the Living care (B1) and medical and nursing care (B2) and social participation(B5) are the most important factors.

Similarly, according to the calculation of the $\mathrm{C}$ layer index with respect to the weight of the A layer, the $\mathrm{C}$ layer of the indicators of the importance of sorting." home sick-bed services (C21)" weight is the biggest, service quality is good or bad primary measure of condition is the ability to ensure the home sick-bed services, followed by daily professional nursing services, postoperative rehabilitation nursing service and Acute disease nursing services.

In the safety and security guarantee layer, we can see that most experts think the services quality is not very good, just "General". The same results also exist in spiritual consolation layer and social participation layer. All these results show that the overall aged care service quality is not so satisfied and still have much space to improve. 


\section{Conclusions}

Using the models developed from AHP and fuzzy comprehensive evaluation, a quantitative evaluation index system for aged community home-care services is constructed in this paper. According to the model, the service quality in 20 communities in Nanchang city was evaluated. The results show that the average level of service quality is between "general" and "good", and that the construction of community home aged care in Nanchang city has achieved remarkable achievement, but there is still an urgent need for a further escalation and improvement. Firstly, according to the model, the currently "living care services" need to continuously improve, especially, the elderly meal services is most import for the aged. Secondly, the medical and nursing services system need to continuously improve. For the currently medical service, it still can not meet the demand to the aged, But as the deterioration of physical function, the higher requirement is raised for medical services. Thirdly, spiritual consolation services are becoming more and more important for the feeling of loneliness is continuously increasing as the age grows and the need for chat service and the like continuously increase.

\section{References}

[1] Xiaodong Yu. Research on Service Quality Evaluation System of Aged Care[D].Tsinghua University(2010).

[2] Jianyi Zuo, Zhihua Zhou. Practice and thought on rural home care services in Ningbo. Ningbo Newsport,10(2013):50-51.

[3] xiaoyi Zhang, Bangcheng Liu. Service Quality of Elderly Homecare in Community:A SERVQUAL-based Structural Model. Chinese Journal of Population Science,3(2011),83-92,112.

[4] Chuhui Liao, Wei Gan, Juan Chen. Research on the evaluation of the Community home aged care service quality in Chinse firt-tier cities. Journal of Zhongnan University of Economics and Law,2(2014):46-50.

[5] N Yang, M Han, S Chen, et al. Research on Evaluation Method Based on Modified Buckley

Decision Making and Bayesian Network. Mathematical Problems in Engineering, 3(2015):27-32.

[6] Shihua Yan. Method of Comparison Matrix Consistency Adjustment Based on AHP. Armament Automation, 4(2008):27-31.

[7] Shubai Xu. Analytic hierarchy process principle. Tianjin:Tianjin University(1988).

[8] Lunbiao Yang. Principle of Fuzzy Mathematics and Applications[M].Guangzhou:South China University of technology press(2000):67-80.

[9]Shiyong Li, Engineering Fuzzy Mathematics and Applications.Harbin:Harbin Institute of Technology press( 2004):101-108. 